\title{
Prognostic Aspects, Survival Rate, and Predisposing Risk Factors in Patients with Fournier's Gangrene and Necrotizing Soft Tissue Infections: Evaluation of Clinical Outcome of 55 Patients
}

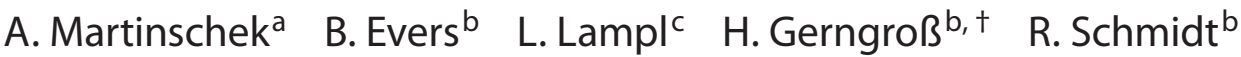 \\ C. Sparwasser ${ }^{a}$ \\ Departments of a Urology, ${ }^{b}$ Surgery and ${ }^{c}$ Anesthesiology and Intensive Care Medicine, Federal Armed Forces \\ Hospital of Ulm, Ulm, Germany
}

\section{Key Words}

Necrotizing soft tissue infection - Fournier's gangrene • Necrotizing fasciitis $\cdot$ Gas gangrene $\cdot$ Hyperbaric oxygen therapy

\section{Abstract}

Objective: To determine predisposing or prognostic factors and mortality rates of patients with Fournier's gangrene compared to other necrotizing soft tissue infections (NSTI). Material and Methods: Data of 55 intensive care patients (1981-2010) with NSTI were evaluated. Data were collected prospectively. Results: $43.4 \%$ of the patients were in septic condition and $27.3 \%$ were hemodynamically unstable. Half of the patients showed predisposing factors (52.7\%). The lower extremity (63.2\%), abdomen (30.9\%), and perineum (14.5\%) were most affected. Polymicrobial infections were frequent $(65.5 \%$, mean 2.8 , range: $1-4)$. The mortality rate was $16.4 \%(n=9)$. An increase was shown for diabetes mellitus (20\%), cardiac insufficiency (22.3\%), septic condition at presentation (33.3\%), abdominal affection (47.1\%), and hemodynamic instability (46.7\%). Comparing survivors and nonsurvivors, statistical significance was seen with age $(p<$ $0.001)$, septic condition at admission $(p<0.001)$, hemody- namic instability $(p<0.001)$, low blood pressure $(p<0.001)$, and abdominal affection $(p<0.001)$. In laboratory findings, an increase of creatine kinase $(p<0.001)$ and lactate $(p<$ $0.001)$ and a decrease of antithrombin III $(p<0.007)$ and the Quick value $(p<0.01)$ proved to be significant. Conclusion: Patients with Fournier's gangrene do not differ in all aspects from those with other NSTI. Successful treatment consists of immediate surgical debridement, broad-spectrum antibiotic treatment, and critical care management. Supportive hyperbaric oxygen therapy should be considered.

Copyright $\odot 2012$ S. Karger AG, Basel

\section{Introduction}

Necrotizing soft tissue infections (NSTIs) constitute a spectrum of diseases of any layer within the soft tissue compartment that is associated with necrotizing changes, characterized by fulminate, widespread necrosis of soft tissue, systemic toxicity, and high mortality. Although no clear definition exists, the disease can clinically be subdi-

This paper is dedicated to Prof. Heinz Gerngroß (July 2, 1947 to June 2, 2005).

\section{KARGER \\ Fax +41 613061234 \\ E-Mail karger@karger.ch}

www.karger.com (c) 2012 S. Karger AG, Basel

0042-1138/12/0892-0173\$38.00/0

Accessible online at:

www.karger.com/uin
Andreas Martinschek, MD

Department of Urology, Federal Armed Forces Hospital of Ulm Oberer Eselsberg 40

DE-89081 Ulm (Germany)

Tel. +49 7311710 2101, E-Mail Martinschek@web.de 
vided in superficial infections (necrotizing cellulitis) confined to the cutis and subcutis, and those reaching deeper regions [1]. Necrotizing fasciitis is a severe NSTI involving primarily the superficial fascia and subcutaneous tissue. In the perineal, genital, and perianal regions, it is often referred to as Fournier's gangrene. Gas gangrene, on the other hand, is caused by Clostridia. Despite advances in antibiotic therapy and intensive medical treatment, these infections still pose a serious challenge. The disease is characterized by a rapid aggravation and the frequent loss of extremities, as well as high mortality rates of up to $76 \%$ [2]. It can appear as a result of a trauma, surgical intervention, an accidental or sometimes minor injury, or even spontaneously. Originally reported by Baurienne [3] in 1794, Jean Alfred Fournier [4], a Parisian dermatologist and venereologist, was the first to describe this fulminate infection of the subcutaneous tissue and superficial fascia to the scrotum, penis, and perineum. The first description of necrotizing fasciitis in a significant number of cases was published by Joseph Jones [5] in 1871, a surgeon in the Confederate army, who reported a mortality rate of $46 \%$ in 2,642 soldiers afflicted during the Civil War. In 1924, Meleny [6] isolated Streptococcus pyogenes as a causative organism of soft tissue infections. Streptococcus pyogenes is the most referred to cause of this disease, named 'flesheating bacteria' or 'killerbug' in the lay press as well as the medical literature [7]; however, more often a synergistic infection of aerobic and facultative anaerobic bacteria can be found [8]. This synergistic action of aerobic and anaerobic organisms plays a major role in the progressive course of the infection [9]. Wilson [10] introduced the term 'necrotizing fasciitis', which is still used today, indicating the necrosis of the fascia as pathognomonic. The infection induces a fulminant inflammatory response which leads to obliterative endarteritis, thrombosis of the cutaneous and subcutaneous vessels, and tissue necrosis. Clostridia infections (gas gangrene) come along with profound toxemia, extensive edema, massive necrosis of tissue, and a variable degree of gas production. The production of alpha-toxin (exotoxin) induces a destruction of the cell membranes, hemolysis, ablation of the fascia, and myonecrosis, and is cardiotoxic as well [11]. Currently, the term 'necrotizing soft tissue infections' summarizes diseases comprising the mere necrosis of the skin as well as life-threatening infections of the fascia and the muscles. For practical purposes, the various diseases are often grouped together due to their similar clinical pattern and principles of treatment [1], comparable prognostic factors, predisposing diseases, and mortality rates. The mortality rate, which reaches $76 \%$, reflects the lethal threat of the disease and the importance of an early diagnosis and optimized therapy as an interdisciplinary challenge. Our research took in consideration the aspects of the predisposing factors, the clinical findings and results of diagnostics, strategies of therapy, and results to discover factors which have an impact on the survival of the patients.

\section{Patients and Methods}

The study included all patients presenting at our hospital with necrotizing infections of the skin and the subcutis with or without necrosis of the muscle which showed a systemic progression and who were in need of intensive care. Patients treated on peripheral wards were not included. Thus, we look at a selected group of patients with an advanced stage of the disease. From 1981 to 2009, the medical records of 55 patients were reviewed for demographics, age, gender, predisposing factors, clinical and laboratory findings, anatomic site of infection, surgical interventions, additional treatment, clinical outcome, and mortality. A statistical evaluation was performed to identify predisposing and prognostic factors. The patient cohort consisted of 34 male patients (61.9\%) and 21 female patients (38.1\%), ranging in age from 14 to 82 years, with a median age of 48 years. Sepsis was diagnosed on the basis of the definitions issued by the American College of Chest Physicians/Society of Critical Care Medicine (ACCP/SCCM) Consensus Conference Committee in 1992 [12], i.e. when: (1) an infection was clinically or microbiologically detected, and (2) a systemic host reaction was present (hypothermia $<36.0^{\circ} \mathrm{C}$ or hyperthermia $>38.0^{\circ} \mathrm{C}$, tachycardia $>90$ beats/min, leukocytosis $>12,000 / 1$ or leukopenia $<4,000 / 1$ or tachypnea $>20$ breaths/min), and infection-induced organ dysfunction was detected [metabolic acidosis $(\mathrm{pH}<7.36)$, acute encephalopathy, thrombocytopenia $(<60,000 / 1)$, arterial hypotension ( $<100 \mathrm{~mm} \mathrm{Hg}$ systolic), or renal dysfunction (serum creatinine $>1.5 \mathrm{mg} / \mathrm{dl}$ or oliguria $<500 \mathrm{ml} /$ day)]. All patients were treated with immediate surgical debridement. Nonviable and infected tissue was excised until healthy tissue was reached. Repeated debridements were performed in cases with worsening clinical parameters, recurrent tissue necrosis, or delayed wound recovery. Broad-spectrum antibiotics were immediately started and later adapted according to microbiological results. Hyperbaric oxygen therapy (HBO) was administered according to the 'Boerema-Schema TS $300-90$ ', i.e. $300 \mathrm{kPa} / 3$ bar for $90 \mathrm{~min}$, while continuing the intensive care [11]. The data were collected prospectively and analyzed retrospectively. A commercially available statistical software package (SPSS Inc., Chicago, Ill., USA) was used for the statistical analyses, performing the Mann-Whitney $U$ test and Fisher's exact test. $\mathrm{p}<0.05$ was considered significant.

\section{Results}

Approximately half (52.7\%) of the patients showed predisposing factors, about a third (32.7\%) of the patients had a cardiac insufficiency NYHA II and higher, and 27.3 and $25.4 \%$ suffered from diabetes mellitus and hypertension, 
Table 1. Comparison of patients with Fournier's gangrene to NSTI in other locations

\begin{tabular}{|c|c|c|c|c|c|c|c|}
\hline & \multicolumn{2}{|l|}{$\begin{array}{l}\text { Total } \\
(\mathrm{n}=55)\end{array}$} & \multicolumn{2}{|c|}{$\begin{array}{l}\text { Fournier's gangrene } \\
(\mathrm{n}=8)\end{array}$} & \multicolumn{2}{|c|}{$\begin{array}{l}\text { Other localizations } \\
(\mathrm{n}=47)\end{array}$} & $\mathrm{p}$ \\
\hline Male/female, $\mathrm{n}$ & $34 / 21$ & & $5 / 3$ & & $29 / 18$ & & n.s. \\
\hline Predisposing factors & $52.7 \%$ & $\mathrm{n}=29$ & $50.0 \%$ & $\mathrm{n}=4$ & $53.2 \%$ & $\mathrm{n}=25$ & n.s. \\
\hline Septic condition & $43.6 \%$ & $\mathrm{n}=24$ & $37.5 \%$ & $\mathrm{n}=3$ & $44.6 \%$ & $\mathrm{n}=21$ & n.s. \\
\hline Hemodynamically unstable & $27.3 \%$ & $\mathrm{n}=15$ & $20.0 \%$ & $\mathrm{n}=2$ & $27.6 \%$ & $\mathrm{n}=13$ & n.s. \\
\hline Muscular affection & $32.7 \%$ & $\mathrm{n}=18$ & $37.5 \%$ & $\mathrm{n}=3$ & $31.9 \%$ & $\mathrm{n}=15$ & n.s. \\
\hline Average stay on ICU, days & 11.8 & 649 & 11.2 & 90 & 11.9 & 559 & n.s. \\
\hline Mortality rate & $16.4 \%$ & $\mathrm{n}=9$ & $12.5 \%$ & $\mathrm{n}=1$ & $17.0 \%$ & $\mathrm{n}=8$ & n.s. \\
\hline
\end{tabular}

respectively. Arteriosclerosis was found in $23.6 \%$. At the time of admission, $52.7 \%$ of the patients received controlled respiration, $43.6 \%$ were already septic, and $27.3 \%$ showed hemodynamic instability, necessitating the application of vasopressors. All patients (100\%) showed local reddening, swelling, and necrosis. All addressable patients ( $\mathrm{n}=17,30.9 \%)$ complained of severe pain. The typical crepitation was found in $73.6 \%$ of all cases. The lower extremities were most frequently affected (63.2\%), followed by the abdominal wall (30.9\%) and the perineum (14.5\%). In the other 11, NSTI was found on the hand $(\mathrm{n}=2)$, fore$\operatorname{arm}(n=3)$, neck $(n=2)$, and thorax $(n=6)$. Two or more areas were affected in 18 cases. Muscular affection could be found in $32.7 \%$ of the cases. The number of bacteria isolated in each patient varied from 1 to 4 species. The most frequent pathogen was Clostidium perfringens (67.3\%), followed by Enterobacter cloacae (30.9\%), Streptococcus group A (30.9\%), and Staphylococcus aureus (25.4\%). Polymicrobial infections were found in $65.5 \%$ of all patients (mean 2.8). The surgical procedures included repeated debridements in $78.2 \%$ and amputations and exarticulations in $25.4 \%$ of the cases (mean number of interventions: 3 ). During intensive care treatment, controlled respiration was necessary in $74.5 \%$ of all cases and the application of vasopressors was necessary in 50.9\%. All patients received combined intravenous antibiotic therapy. The average stay on the intensive care unit was 11.8 days. Supportive HBO was performed in nearly all cases (96.4\%). As the hyperbaric chamber is included in our intensive care unit, unproblematic continuation of intensive care treatment was possible. The average number of hyperbaric oxygen treatments was 7.5. Our mortality rate was $16.4 \%(n=9)$. An increase in the mortality rate could be seen in patients with diabetes mellitus (20\%), cardiac insufficiency (22.3\%), a septic condition at presentation (33.3\%), muscular involvement (38.9\%), and above all if the abdomen was affected

Fournier's Gangrene and Necrotizing Soft Tissue Infections
(47.1\%) or the disease had already caused hemodynamic instability (46.7\%). Taking into account changes in intensive care management and available antibiotics, we divided the cohort into three decade groups (1981-1990, 19912000 , and 2001-2010). We found an improvement in mortality rates of $20 \%(3 / 15), 15.8 \%(3 / 19)$, and $14.3 \%(3 / 21)$.

The comparison between the survivors and the nonsurvivors shows a statistical significance for the factor age (43.7 vs. 64.6 years, $\mathrm{p}<0.001$ ), a septic condition at presentation ( 88.9 vs. $34.8 \%, \mathrm{p}<0.001)$, hemodynamic instability (77.8 vs. $17.4 \%, \mathrm{p}<0.001)$, low systolic blood pressure (92.8 vs. $120.8 \mathrm{~mm} \mathrm{Hg}, \mathrm{p}<0.001$ ), abdominal affection $(88.9$ vs. $19.6 \%, \mathrm{p}<0.001)$, and muscular involvement (77.8 vs. $4.34 \%, \mathrm{p}<0.001$ ).

The increase of creatine kinase (383 U/l vs. $501 \mathrm{U} / \mathrm{l}$, $\mathrm{p}<0.001)$ and lactate $(2.2 \mathrm{vs} .10 .7 \mathrm{mmol} / \mathrm{l}, \mathrm{p}<0.001)$ as well as a decrease of the antithrombin III parameter $(62.1$ vs. $47.6 \%, \mathrm{p}<0.007$ ) and the Quick value (73.3 vs. $47.6 \%$, $\mathrm{p}<0.01$; corresponding to the INR) at time of admission also proved to be a significant factor for a fatal prognosis.

The cohort included 8 patients with Fournier's gangrene (male-to-female ratio 5:3) in need of intensive care medicine. Additional to the scrotal/penile infection, an affection of the perineum was found in all of these patients. Muscular involvement could be found in 3 patients. One patient (with muscular affection) in this group died. Patients with Fournier's gangrene did not show significant differences concerning any item compared to other localizations of NSTI (table 1).

\section{Discussion}

Severe NSTIs are life-threatening diseases with high mortality rates of $16.9-34 \%$ in larger clinical studies and even reaching $76 \%$ in smaller studies $[2,9]$. An incidence 
of $0.4 / 100,000$ inhabitants has been reported [13]; in the USA the incidence has been determined to be 0.04 cases per 1,000 person-years in a larger study, using insurance databases from various states [11]. When Stephens et al. [14] specifically reviewed Fournier's gangrene in 1993, 449 cases were reported from 1979 to 1988 , giving a mean of 45 cases per year. Since then from 1989 to 1998, 973 cases have been reported in the English literature, giving a mean yearly incidence of 97 cases [15]. Death is usually not caused by the often extensive tissue defect, but by the systemic effects of severe sepsis, coagulopathy, acute renal failure, diabetic cetoacidosis, adult respiratory distress syndrome, mesenteric ischemia, aspirations pneumonia, myocardial infarction, hepatic failure, bone marrow failure, and adrenal insufficiency, leading to multiorgan failure $[2,8,9]$. Untreated, the mortality of gas gangrene approaches $100 \%$; surgical treatment alone may reduce the rate to $38 \%$ and a combination of all therapeutic options (surgical debridement, antibiotics, critical care medicine, and HBO) may reduce it to $13-19 \%[11,16]$. Without surgical treatment, necrotizing fasciitis has mortality rates of $100 \%$ [13], and overall mortality rates of about 30\% [8]. McHenry et al. [2] showed a cumulative mortality rate in nearly 700 patients with Fournier's gangrene of $34 \%$. In smaller studies, a mortality rate of $7.5-40 \%$ was reported $[9,16,17]$. In the biggest study with 1,726 reviewed cases, Eke [15] stated that the mortality rate associated with Fournier's gangrene is lower than that of other forms of necrotizing fasciitis, varying from 3 to $45 \%$, with an overall mortality of $16 \%$. In our study, we achieved a mortality rate of $16.4 \%$ by combining aggressive surgical debridement, antibiotics, critical care management, and $\mathrm{HBO}$ in a selected group of patients in an advanced stage, and even reducing it to $14.3 \%$ in the last decade.

Early diagnosis of NSTI requires a high level of suspicion and familiarity with the clinical findings [16]. Establishing the diagnosis at the earliest time-point presents the major challenge [17]. Typical clinical findings are severe painful swelling, erythema, and blisters [8]. Sometimes only pain, tenderness, and local hyperthermia may initially be observed, making it difficult to distinguish NSTI from cellulitis [1]. Once the infection progresses, the more typical signs and symptoms including tense edema beyond the area of compromised skin, skin discoloration, blisters/bullae, or crepitus can be identified [1, 18]. As the infection spreads along the fascial planes and deeper areas, painless black ulcers may appear. With progressive infection, vascular occlusion, ischemia, and tissue necrosis may occur. As a result, superficial nerves may be damaged, producing localized anesthesia [1] be- fore patients complain about pain [8]. Symptoms may develop over a period of a few hours, but the process can also take several days. Disproportional pain should especially alert the clinician [1].

All of our patients showed typical symptoms. A crepitation of the soft tissue could be perceived in the majority of the cases. The bacterial synergism involves the production of exotoxins whose activities result in necrosis and synthesis of gases that produce the repulsive stench and crepitus, pathognomonic of anaerobic infection [15]. The finding of crepitus and soft tissue air on plain radiography are pathognomonic, although these signs are present in only 37 and 57\%, respectively [19]. The localizations of NSTI in our study are comparable to others [20]. We found involvement of the abdominal wall to be a significant factor for mortality.

In a study of Heitmann et al. [8], 4-6 patients with involvement of the trunk died, but none with peripheral affection. Pessa and Howard [18] also state that affection of the abdominal wall leads to higher mortality rates. Czymek et al. [9], however, found no statistical relationship between the involvement of a particular anatomical region and the need for surgical procedures. Additionally, the percentage of body surface affected failed to be a statistically significant parameter [2,21]. Although NSTI may affect all ages and both sexes, most studies, as well as our results, show a preference of male sex and a median age of about $48-59$ years $[2,4,5,13,22]$. Increased age has been shown to be a statistically significant factor for the mortality of patients in the studies of McHenry, Eke and Sorensen, Unalp (age $>60$ years) and Yanar (age $>55$ years), which is in accordance with our results $[2,9$, $14,15,21]$. Gender does not seem to influence the prognosis, as stated before in other studies [22].

Various pre-existing conditions are associated with an increased risk of NSTI. In the literature, diabetes mellitus, chronic alcohol abuse, peripheral vascular disease [2, 16], chronic renal failure, hepatic dysfunction [15, 22], heart disease, acquired immunodeficiency syndrome, malignant disease $[1,16,23]$, nonsteroidal anti-inflammatory drugs [22], drug abuse (specifically injection of drugs), trauma, decubitus ulcer, immune impairment [21], chronic obstructive lung disease, cortisone use [16], obesity, and malnutrition [2] have been reported as risk factors. In our study, $52.7 \%$ of the patients showed comorbidities. McHenry et al. [2] stated that the presence of peripheral vascular disease was a factor that approached statistical significance in the patients who died from NSTI. In the study of Czymek et al. [9], all patients who died had pre-existing cardiac conditions (chronic cardiac 
insufficiency, coronary heart disease, arterial hypertension). Yanar et al. [16] reported cardiac disorders in 31\% of his patients. Sorensen et al. [24] found congestive heart failure and hypertension to be a significant mortality predictor.

Diabetes mellitus was seen in $27.3 \%$ of our patients and was associated with a higher mortality rate. This has been confirmed by other studies. Unalp found diabetes mellitus as the most common predisposing factor in patients with Fournier's gangrene (35.3\% of 68 patients), associated with a significantly higher mortality rate (29.1 vs. 10.3\%) [22]. Diabetes mellitus clearly is a risk factor for NSTI with a reported prevalence of $39-64 \%$ in the published literature $[9,16,25,26]$. Furthermore, the presence of diabetes has an adverse effect on the survival of the patient [20]. Renal failure and coagulopathy were also significantly associated with higher mortality rates [24].

The existence of one or more comorbidities should raise the physicians' suspicion of NSTI. Our patients presented with signs of sepsis in $43.4 \%$, and in a hemodynamically unstable condition $27.3 \%$; mechanical ventilation was necessary in $52.7 \%$. Faucher et al. [17] reported similar rates (46\% septic shock, $47 \%$ mechanical ventilation), and most other studies showed a lower percentage of critically ill patients [14, 21]. Like others, we found that a septic condition and hypotension at the time of admission were significant predicting factors for mortality and outcome [14, 16, 21, 27]. Furthermore, our study shows that an increase of creatine kinase and lactate parameters, as well as a decrease of antithrombin III parameter and the Quick value (corresponds to INR), are significant parameters for predicting an unfavorable outcome. In the literature, systemic acidosis, low hematocrit and albumin levels, high serum creatinine, elevated alkaline phosphatase [21], and elevated urea [20,21] were also significantly related to mortality.

NSTI requires a combined therapy consisting of early operative debridement, intensive care medicine, and combined antibiotic therapy. In 1981, Kaiser and Cerra [23] published their results with immediate surgical excision (3-4 h) of all necrotic tissue, antibiotics, aggressive nutritional support, and early skin coverage leading to a mortality rate of $8.3 \%$, whereas treatment with antibiotics and delayed (1-3 days) excision of necrotic tissue was associated with a mortality rate of $75 \%$.

The fascial structures of the pelvic floor and the anogenital region are of major importance for understanding the spread of Fournier's gangrene in the pelvic floor and anogenital region. Colles' fascia, which is the superficial fascia of the perineum, is continued as tunica dartos over the scrotum and penis. Colles' fascia is attached to the urogenital diaphragm and merges with Scarpa's fascia of the anterior abdominal wall. Buck's fascia surrounds the deeper structures of the penis and periurethral region. Infection may spread from Buck's fascia to Colles' fascia and the dartos fascia especially in cases of injury [9]. When the penis is involved, the corpora are usually spared while the skin sloughs off. However, synchronous thrombosis of the corpus spongiosum and cavernosum has been reported $[15,28]$. The male gonads are relatively well protected since they are covered by a special fascia (tunica vaginalis) and receive their blood supply from the retroperitoneum. Orchidectomy is therefore necessary only in rare cases [9]. In one study, orchidectomy was performed, at the discretion of the surgeon, in 6 patients because of the observed severe infection in peritesticular tissues; however, in the pathological review of the specimen, the testicles were not found to be involved [16]. On the other hand, a necrotic testis should always raise the suspicion of a retroperitoneal or intra-abdominal spread of the disease [9].

The mean number of debridements varies from 2 to 8 surgical procedures per patient, with absolute numbers ranging from 1 to 21 ; for a control of infection most studies report about 2-3 surgical measures [5, 13, 22]. Several studies failed to find a correlation between the number of debridements and the rate of mortality $[15,21]$. As Faucher et al. [17] stated, initial surgery should be a true 'search and destroy' mission, in which all necrotic and infected tissue is resected, regardless of the wound that is created and the potential problems of complete closure, and samples should be taken for microbiologic culture [16]. Mere incision and drainage does not constitute adequate therapy [8].

Various bacteria have been identified in patients with NSTIs. The organisms are in most cases indigenous commensals and include Clostridia, Klebsiella, streptococci, coliforms, staphylococci, bacteroids, and corynebacteria. In synergism, one bacterium produces a nutrient for another, which in turn produces a leukocidal toxin, protecting both from phagocytosis. Furthermore, the aerobe produces a thriving atmosphere for the anaerobe by decreasing the oxygen tissue tension [14-16]. Fungal infections may be found, but they are rare. In our study, $65 \%$ of the cases were polymicrobial and 35\% were monobacterial. Clostridium perfringens (67.3\%), Streptococcus group A (30.9\%) and Staphylococcus aureus (25.4\%) were the most common microorganisms identified in our microbiological cultures. 
Broad-spectrum antimicrobial therapy should be started empirically at once but should not delay surgical treatment as there is no proof that antibiotics can reduce the extent of surgical debridement or even stop NSTIs [1, 4]. Empirical antibiotic therapy should be a triple drug therapy, covering a polymicrobial infection with Grampositive, Gram-negative, and anaerobic organisms $[1,5$, 19]. Penicillin is recommended for streptococci, metronidazole for anaerobic organisms, and a third-generation cephalosporin (with or without gentamicin) for coliform organisms and staphylococci [15]. Combinations of penicillin (or ampicillin or third-generation cephalosporins), metronidazole, and aminoglycosides for Gram-negative aerobes have been reported $[4,5]$.

When the infection is acquired in the hospital, multiresistant bacteria should be taken into account. The antibiotic regimen must be reassessed based on the microbiological results. Antibiotics should be continued until no further debridement is needed and the patients' condition has improved, but no specific guideline exists as to duration of treatment. Long-term administration is not necessary and may predispose the patient to wound colonization with drug-resistant organisms $[1,25]$.

Physiologic support including mechanical ventilation combined with close invasive monitoring in an intensive care unit setting has to be established. Organ failure, such as acute renal failure and acute respiratory distress syndrome, requires replacement therapies. Appropriate early nutritional support, delivered enterally if possible, helps to control the catabolic response of these patients. Aggressive fluid resuscitation and blood component therapy is often required during the perioperative period. Judicious control of glucose, as well as novel therapeutic approaches for severe sepsis and septic shock, should be considered to optimize the host response to infection [19].

As a local infection progresses, the bacterial metabolism depletes the oxygen in the affected area. Additionally, the accumulation of metabolic products lowers the oxidation-reduction potential and tissue oxygen levels. This leads to an inhibition of phagocytosis and bacterial clearance, a decrease of fibrocytic synthesis of collagen, and a reduced effect of aminoglycosides [11,26].

$\mathrm{HBO}$ has several beneficial effects on severe NSTI, especially on clostridial infections. HBO can raise the physically dissolved oxygen level in blood from $0.3 \mathrm{ml} \mathrm{O} / \mathrm{dl}$ (normal atmospheric pressure) to $2.1 \mathrm{ml} / \mathrm{dl}$ (1 bar), $4.4 \mathrm{ml} /$ $\mathrm{dl}$ (2 bar), and $6.8 \mathrm{ml} / \mathrm{dl}$ (3 bar) [29]. $\mathrm{A} \mathrm{pO}_{2}$ of $80-250 \mathrm{~mm}$ $\mathrm{Hg}$ stops the production of alpha-toxin and a $\mathrm{pO}_{2}$ of 1,500 $\mathrm{mm} \mathrm{Hg}$ is bactericide to Clostridia [11]. Therefore, $\mathrm{HBO}$ may inhibit bacterial reproduction, thus enabling the body to utilize its own host defense mechanisms. The systemic toxicity as well as the local spread of the necrosis can be reduced [28], which will reduce the probability of amputations and therefore the grade of handicap [11, 29], thus leading to better mortality rates [25].

In NSTI, the use of HBO remains disputable $[8,30]$. Available data are controversial, and although the beneficial effects have been reported in the literature $[9,26]$, there is increasing doubt as to whether $\mathrm{HBO}$ has a measureable positive effect on morbidity and mortality $[9,27,31,32]$. A review of 1,726 cases of Fournier's gangrene concludes that hyperbaric oxygenation increases tissue oxygen tension to a level that inhibits and kills anaerobic bacteria, while also reducing systemic toxicity, limiting necrosis and enhancing demarcation of gangrene [15]. Riseman et al. [30] report of a significant reduction of mortality as well as the number of debridements due to application of hyperbaric oxygen. Korhonen et al. [33] showed in a study of patients with Fournier's gangrene $(\mathrm{n}=33)$ a very low $(9 \%)$ mortality rate with $\mathrm{HBO}$. In a retrospective analysis comparing patients of two clinical centers ( 48 with $\mathrm{HBO}$ vs. 30 without HBO) George et al. [32] reported no significant differences in the number of debridements, length of stay at the hospital, or duration of antibiotic therapy. Although not statistically significant, they observed a mortality rate of $13.3 \%$ in the non- $\mathrm{HBO}$ group and $8.3 \%$ in the $\mathrm{HBO}$ group [31]. In conclusion, $\mathrm{HBO}$ is no standard therapy but may be an important therapeutic adjunct in NSTI $[11,25]$.

Several scores have been published in order to predict mortality in NSTI. Wong et al. [34] created the Laboratory Risk Indicator for Necrotizing Fasciitis Score (LRINEC), identifying six independent variables associated with NSTI. Each variable, if present, gives a specific number of points towards the final score, ranging from 0 to 13 , with a probability of $<50 \%$ for NSTI in 5 or less points, between 50 and $75 \%$ for $6-7$ points, and more than $75 \%$ if the score is 8 or more points. For the last two groups, they showed a PPV of $92 \%$ and an NPV of $96 \%$. The Fournier's Gangrene Severity Index (FGSI) is a numeral score to describe the acuity of the disease. Several studies proved a higher mortality rate for FGSI score $>9$ [24]. Hospital stay ranged 2-278 days in the biggest retrospective study of 1,726 cases [15]. Sorensen et al. [24] calculated median hospital charges for Fournier's gangrene patients to USD 27,646 (survivors USD 26,574 vs. nonsurvivors USD 40,871). Faucher et al. [17] reported mean charges of USD 153,803 for survivors (mean hospital stay 26.3 days). Thirty percent of survivors required ongoing care after hospital discharge, most often for open wound closure [24]. 


\section{Conclusion}

Most importantly, early diagnosis of NSTI requires a high level of suspicion. The relative rarity of NSTI, coupled with its frequently subtle initial presentation, often presents a major diagnostical challenge. It is generally agreed that the successful therapy of severe NSTI consists of several equally important cornerstones: immediate adequate surgical debridement, intravenous antibiotic treatment (for example acylureido-penicillin + aminoglycoside + metronidazole, or carbapenem in case of an allergy to penicillin), and modern critical care management. The fourth pillar of treatment may be supportive $\mathrm{HBO}$ in infections with Clostridia; in other severe NSTIs, $\mathrm{HBO}$ should be taken into account as an additional therapeutic procedure.

\section{References}

1 Cainzos M, Gonzalez-Rodriguez FJ: Necrotizing soft tissue infections. Curr Opin Crit Care 2007:13:433-439.

2 McHenry CR, Piotrowski JJ, Petrinic D, Malangoni MA: Determinants of mortality for necrotizing soft-tissue infections. Ann Surg 1995;221:558-563, discussion 63-65.

3 Baurienne H: Sur une plaie contuse qui s'est terminée par la sphacele de la scrotum. J Med Chir Pharm 1794;20:251-256.

4 Fournier J: Gangrene foudroyante de la verge. Med Pract 1883;4:589-597.

5 Jones J: Investigation upon Nature, Causes and Treatment of Hospital Gangrene as It Prevailed in the Confederate Armies 18611865. Surgical Memories of the War of Rebellion. New York, US Sanitary Commission, 1871.

6 Meleny F: Hemolytic streptococcal gangrene. Arch Surg 1924;9:317-331.

7 Moses AE: Necrotizing fasciitis: flesh-eating microbes. Isr J Med Sci 1996;32:781-784.

8 Heitmann C, Pelzer M, Bickert B, Menke H, Germann G: Surgical concepts and results in necrotizing fasciitis (in German). Chirurg 2001;72:168-173.

9 Czymek R, Hildebrand P, Kleemann M, et al: New insights into the epidemiology and etiology of Fournier's gangrene: a review of 33 patients. Infection 2009;37:306-312.

10 Wilson B: Necrotizing fasciitis. Am Surg 1952;18:416-431.

11 Lampl L, Frey G, Fischer D, Fischer S: Hyperbaric oxygenation: utility in intensive therapy - part 2 (in German). Anasthesiol Intensivmed Notfallmed Schmerzther 2009;44: 652-658.

12 Bone RC, Sibbald WJ, Sprung CL: The ACCP-SCCM consensus conference on sepsis and organ failure. Chest 1992;101: 1481-1483.

13 Kaul R, McGeer A, Low DE, Green K, Schwartz B: Population-based surveillance for group A streptococcal necrotizing fasciitis: clinical features, prognostic indicators, and microbiologic analysis of seventy-seven cases. Ontario Group A Streptococcal Study. Am J Med 1997;103:18-24.
14 Stephens BJ, Lathrop JC, Rice WT, Gruenberg JC: Fournier's gangrene: historic (17641978) versus contemporary (1979-1988) differences in etiology and clinical importance. Am Surg 1993;59:149-154.

15 Eke N: Fournier's gangrene: a review of 1726 cases. Br J Surg 2000;87:718-728.

16 Yanar H, Taviloglu K, Ertekin C, et al: Fournier's gangrene: risk factors and strategies for management. World J Surg 2006;30: 1750-1754.

17 Faucher LD, Morris SE, Edelman LS, Saffle JR: Burn center management of necrotizing soft-tissue surgical infections in unburned patients. Am J Surg 2001;182:563-569.

18 Pessa ME, Howard RJ: Necrotizing fasciitis. Surg Gynecol Obstet 1985;161:357-361.

19 Anaya DA, Dellinger EP: Necrotizing softtissue infection: diagnosis and management. Clin Infect Dis 2007;44:705-710.

20 Singh G, Sinha SK, Adhikary S, Babu KS, Ray P, Khanna SK: Necrotising infections of soft tissues - a clinical profile. Eur J Surg 2002; 168:366-371.

21 Mulla ZD, Gibbs SG, Aronoff DM: Correlates of length of stay, cost of care, and mortality among patients hospitalized for necrotizing fasciitis. Epidemiol Infect 2007;135: 868-876.

22 Kihiczak GG, Schwartz RA, Kapila R: Necrotizing fasciitis: a deadly infection. J Eur Acad Dermatol Venereol 2006;20:365-369.

23 Kaiser RE, Cerra FB: Progressive necrotizing surgical infections - a unified approach. J Trauma 1981;21:349-355.

24 Sorensen MD, Krieger JN, Rivara FP, Klein $\mathrm{MB}$, Wessells H: Fournier's gangrene: management and mortality predictors in a population based study. J Urol 2009;182:27422747.
25 Hirn M: Hyperbaric oxygen in the treatment of gas gangrene and perineal necrotizing fasciitis. A clinical and experimental study. Eur J Surg Suppl 1993;570:1-36.

-26 Pizzorno R, Bonini F, Donelli A, Stubinski R, Medica M, Carmignani G: Hyperbaric oxygen therapy in the treatment of Fournier's disease in 11 male patients. J Urol 1997;158: 837-840.

27 Dahm P, Roland FH, Vaslef SN, et al: Outcome analysis in patients with primary necrotizing fasciitis of the male genitalia. Urology 2000;56:31-35, discussion 5-6.

28 Brown DR, Davis NL, Lepawsky M, Cunningham J, Kortbeek J: A multicenter review of the treatment of major truncal necrotizing infections with and without hyperbaric oxygen therapy. Am J Surg 1994;167:485-489.

29 Frey G, Lampl L, Radermacher P, Bock KH: Hyperbaric oxygenation. An area for the anesthetist (in German)? Anaesthesist 1998; 47:269-289.

30 Riseman JA, Zamboni WA, Curtis A, Graham DR, Konrad HR, Ross DS: Hyperbaric oxygen therapy for necrotizing fasciitis reduces mortality and the need for debridements. Surgery 1990;108:847-850.

-31 Mindrup SR, Kealey GP, Fallon B: Hyperbaric oxygen for the treatment of Fournier's gangrene. J Urol 2005;173:1975-1977.

32 George ME, Rueth NM, Skarda DE, Chipman JG, Quickel RR, Beilman GJ: Hyperbaric oxygen does not improve outcome in patients with necrotizing soft tissue infection. Surg Infect (Larchmt) 2009;10:21-28.

33 Korhonen K, Klossner J, Hirn M, Niinikoski $\mathrm{J}$ : Management of clostridial gas gangrene and the role of hyperbaric oxygen. Ann Chir Gynaecol 1999;88:139-142.

34 Wong CH, Khin LW, Heng KS, Tan KC, Low CO: The LRINEC (Laboratory Risk Indicator for Necrotizing Fasciitis) score: a tool for distinguishing necrotizing fasciitis from other soft tissue infection. Crit Care Med 2004;32:1535-1541. 